\title{
BMJ Open Association between cytokine levels, sepsis severity and clinical outcomes in sepsis: a quantitative systematic review protocol
}

\author{
Amal Gharamti (D , ,,2 Omar Samara, ${ }^{3}$ Anthony Monzon, ${ }^{3}$ Sias Scherger, ${ }^{4}$ \\ Kristen DeSanto, ${ }^{5}$ Stefan Sillau, ${ }^{6}$ Carlos Franco-Paredes (D) ,,7 \\ Andrés Henao-Martínez (i) , ${ }^{4}$ Leland Shapiro (i) ${ }^{4,8}$
}

To cite: Gharamti A, Samara 0, Monzon A, et al. Association between cytokine levels, sepsis severity and clinical outcomes in sepsis: a quantitative systematic review protocol. BMJ Open 2021;11:e048476. doi:10.1136/ bmjopen-2020-048476

- Prepublication history and additional supplemental material for this paper are available online. To view these files, please visit the journal online. (http://dx.doi.org/10.1136/ bmjopen-2020-048476).

Received 28 December 2020 Accepted 26 July 2021

\section{Check for updates}

(c) Author(s) (or their employer(s)) 2021. Re-use permitted under CC BY-NC. No commercial re-use. See rights and permissions. Published by BMJ.

For numbered affiliations see end of article.

Correspondence to Dr Andrés Henao-Martínez; andres.henaomartinez@ cuanschutz.edu

\section{ABSTRACT}

Introduction It is widely assumed that sepsis is a life-threatening systemic inflammation caused by a dysregulated host response to infection mediated by an increase in multiple proinflammatory cytokines. The levels of key proinflammatory cytokines tumour necrosis factor, interleukin- $1 \beta$ and interferon $\gamma$ are poorly characterised during sepsis. We believe this project will produce a 'goldstandard' document to which other reports on cytokine levels will be compared. The objective of this systematic review will be to identify key cytokine circulating levels in patients with sepsis and assess the association between these levels and morbidity and mortality outcomes related to sepsis.

Methods and analysis We would include reports of any design except for case reports. Sepsis patients will comprise those with a diagnosis of sepsis, severe sepsis or septic shock. The primary exposure is levels of three proinflammatory cytokines. The primary outcome is mortality at 28 or 30 days. Study subjects can be of any age, sex or ethnicity. Studies will be restricted to the English language. Medline, Embase, Cochrane Library and Web of Science Core Collection will be searched for eligible studies. A database search will include studies from 1985 to May 2020. Two reviewers will independently screen and select studies, assess methodological quality and extract data. A meta-analysis will be performed, if possible, and the Grading of Recommendations Assessment Development and Evaluation Summary of Findings presented.

Ethics and dissemination Formal ethical approval is not required as data will be extracted from existing literature. This systematic review will be disseminated through a peer-reviewed publication and at conference meetings. PROSPERO registration number CRD42020179800.

\section{INTRODUCTION}

Sepsis is an important global health problem associated with significant morbidity and mortality. In 2017, there were an estimated 48.9 million cases of sepsis and 11 million sepsis-related deaths. Approximately 19.7\% of all deaths recorded in 2017 were attributed

\section{Strengths and limitations of this study}

- This study will systematically assess the levels of key cytokines in sepsis patients and study the relationship between these levels and sepsis outcomes such as organ dysfunction and mortality.

- A systematic review of this topic will contribute to a better understanding of the pathophysiology of sepsis and inform therapeutic approaches to anticytokine therapy in patients with sepsis.

- A possible publication bias might limit this study as we will include only peer-reviewed published data. Therefore, this systematic review might not capture evidence from other sources.

to sepsis. ${ }^{1}$ In the USA, in 2013, the annual cost of sepsis was estimated at US\$23.7 billion, representing the largest expenditure for any disease treated in US hospitals. ${ }^{2}$ In 1992, sepsis was defined as a systemic inflammatory response syndrome in the presence of a documented or clinically suspected infection. ${ }^{3}$ In 2016, sepsis-3 diagnostic criteria were introduced, and sepsis was defined as 'life-threatening organ dysfunction due to a dysregulated host response to infection'. SEPSIS-3 defined SEPSIS as an increase in Sequential Organ Failure Assessment (SOFA) score by 2 or more points. Before SEPSIS-3, severe sepsis was defined as sepsis with associated dysfunction of one or more organ systems. Septic shock is a subset of severe sepsis in which hypotension refractory to fluid replacement is present. At present, there is no standard diagnostic clinical or laboratory test for sepsis. ${ }^{4}$ There is considerable variability in the reported levels of key cytokines involved in the pathogenesis of sepsis. Explanations for variability likely include different patient populations, different stages of sepsis (sepsis, severe sepsis or septic shock), type of sample 
used (whole blood, plasma or serum) and methods used to quantify cytokines.

The primary therapies for sepsis remain intravenous fluids, antibiotics and vasopressors. Treatment includes general supportive care that can include ventilatory support with supplemental oxygen or mechanical ventilation and renal replacement therapy (RRT). It is postulated that targeting proinflammatory mediators would reduce mortality and morbidity of sepsis. ${ }^{56}$ However, no specific treatment targeting these cytokines during sepsis has been proven to be effective in clinical trials. ${ }^{7}$

Current concepts of sepsis pathogenesis focus on an excessive host inflammatory response to infection that is mediated by elevated cytokine levels, also known as 'cytokine storm' or 'cytokine release syndrome'. The molecular pathway that leads to a 'cytokine storm' is vague. ${ }^{8}$ The term 'cytokine storm' appears to be first coined in a paper on graft-versus-host disease. ${ }^{89}$ In the early 2000 s, this term was used in articles on infectious diseases. ${ }^{8} 1011$ Pivotal mediators of a cytokine storm include tumour necrosis factor alpha (TNF), interleukin-1 (IL-1) $\beta$ and interferon-gamma (IFN- $\gamma$ ) . The cytokine storm is thought to cause organ malfunction and mortality that accompany sepsis. ${ }^{8}$ Despite extensive study of cytokines in sepsis, the magnitude of cytokine levels and their effects on morbidity and mortality are poorly characterised. TNF, IL-1 $\beta$ and IFN $\gamma$ cytokines have been best characterised as proinflammatory and are thought to drive the hyperinflammatory response to infection. This is underscored by the large number of clinical trials designed to specifically block these cytokines using selective inhibitors of TNF and IL-1 $\beta .{ }^{12}{ }^{13}$ By our count, there have been at least nine late-stage clinical trials using selective TNF blocking drugs and three late-stage studies using IL-1 $\beta$ blockers. This does not include numerous studies (at least 10 studies) where cytokines, in general, are inhibited using drugs like corticosteroids or endotoxin inhibitors (where inhibition will include TNF, IL-1 $\beta$ and IFN $\gamma$ ). Given the large number of resources expended to block these proinflammatory molecules, it is remarkable that no one has published an overall assessment of the levels of these molecules that are present during sepsis. If you plan to block the activity of molecules (cytokines) that are thought to cause disease, it stands to reason you want to know how much of the molecules are present during the disease. If the putative cytokines that cause sepsis are present in unexpectedly low or surprisingly elevated concentrations, this is important information for understanding the cause of sepsis and for designing potential therapies. Moreover, there has been a spectacular failure of sepsis therapies designed to block cytokine activity. As stated by PE Marik '>100 phase 2 or 3 clinical trials of sepsis therapies have failed over the past three decades'. ${ }^{14}$ While not all of these trials directly targeted cytokine suppression, at least 47 clinical trials have targeted cytokine suppression in sepsis; all have failed. These observations underscore the importance of an improved understanding of what constitutes the goal of cytokine-blocking therapy; there is a need to know how many cytokines should be neutralised. This knowledge will inform both clinical and animal studies of sepsis and will help guide in vitro cytokine-blocking experiments.

If the cytokine storm concept of sepsis organ damage is correct, it is reasonable to assume that dysfunction of lungs (a requirement for mechanical ventilation) or kidneys (need for RRT) associates with increased pro-inflammatory cytokine levels compared with sepsis patients without these organ failures. Inhibitors of TNF and IL-1 $\beta$ are used clinically to treat disease with hyperinflammation as a component of pathogenesis (ex, rheumatoid arthritis). These anticytokine therapies carry 'black box' warnings alerting prescribers that they increase the risk for and severity of infections. This documented association between cytokine inhibition and supervening infections suggests we should assess inflammatory cytokine levels for association with secondary infections (infections over and above the proven or presumed infection that caused sepsis). It is conjectured that lower cytokine levels in the circulation will correlate with an increased risk for secondary supervening infections. As well, it is expected that increased concentrations of the natural cytokine inhibitors TNFsRp55, TNFsRp75 or IL-1ra will associate with increased risk for secondary infections

No published or ongoing meta-analyses or systematic reviews evaluating key cytokine levels in sepsis patients and assessing the relationship between cytokine levels and sepsis outcomes were identified. The objective of this systematic review is to characterise levels of key cytokines in the circulation in patients with sepsis and assess the association between these levels and the morbidity and mortality outcomes related to sepsis.

\section{METHODS AND ANALYSIS}

This protocol adheres to the Preferred Reporting Items for Systematic Reviews and Meta-Analyses Protocols (PRISMA) checklist. We will follow the PRISMA guidelines in the development of this systematic review. ${ }^{1516}$

\section{Research questions}

The systematic review will aim to answer the following questions:

1. What are the levels of key pro-inflammatory cytokines in sepsis?

2. How do these levels correlate with sepsis outcomes such as degree of sepsis (sepsis, severe sepsis or septic shock), mortality, organ malfunction and secondary infections?

\section{Eligibility criteria}

\section{Inclusion criteria and exclusion criteria}

Articles will be evaluated according to the criteria described below.

\section{Study design}

This review will consider any design that reports relevant cytokine levels in patient cohorts. This review will 
consider longitudinal studies, prospective cohort studies, randomised and non-randomised clinical trials, casecontrol studies and time-series studies. Case series and descriptive cross-sectional studies will also be included depending on the outcome measured. We will exclude case reports to minimise publication bias. Only studies published in English will be included. The studies must include cytokine concentrations in venous blood serum or plasma and express in mass units per volume. Quantified data depicted only in the graphical form will not be used. The assays used can be ELISA or immunological/ magnetic bead technologies. Studies that measured cytokines by a method other than ELISA or Immunologic/ magnetic bead technologies will be excluded. We will include studies that defined sepsis according to the diagnostic criteria proposed by the American College of Chest Physicians and the Society of Critical Care Medicine in 1992 as the presence of systemic inflammatory response syndrome and infection. The 1992 definition was used instead of current definitions since most studies reporting cytokine levels used the 1992 definition. Participant studies subjects can be of any age, ethnicity or sex. Sepsis studies criteria will include sepsis, severe sepsis, septic shock, purpura fulminans or infection-associated disseminated intravascular coagulation (DIC) (a variant of organ malfunction involving coagulation system failure).

\section{Exposure}

The exposure of interest is the level of proinflammatory cytokines (TNF, IL-1 $\beta$ and IFN $\gamma$ ) in patients with sepsis, severe sepsis or septic shock. The levels of TNF, IL-1 $\beta$ or IFNY are assessed at presentation $(\mathrm{t}=0)$ or admission. We will assess the trend of cytokine levels after admission if such data are available. The cytokines are measured in venous blood (serum or plasma) using ELISA or immunological/magnetic bead technologies (Immunex, others). We will extract the crude unadjusted mean and SD from each cytokine in all studies. We will then calculate the $\mathrm{SE}$ and $\log$-transformed the values. We will enter the logtransform mean and standard errors in a random-effect meta-analysis to combine the estimated log pooled values, which will be back-transformed for interpretation.

\section{Outcomes}

The review will include the following outcome measures: Primary outcome:

1. The mortality rate at 28 or 30 days. Secondary outcomes:

1. Association with global organ dysfunction: SOFA score.

2. Need for supplemental oxygen or mechanical ventilation.

3. Need for RRT.

4. The emergence of secondary or supervening infections.

\section{Search methods}

Search strategy

The search strategy will include a combination of controlled vocabulary terms (specific to each database) and keyword terms. We will search for concepts including sepsis and cytokine levels of TNF, IL-1 $\beta$ or IFN $\gamma$. Terms related to certain inclusion criteria, such as infectionassociated DIC, are not included in the search strategy to maximise recall but will be factored in during fulltext review. We did not include 'cytokine' as a keyword because it is not specific, and we are more interested in the three pro-inflammatory cytokines (TNF, IL-1 $\beta$ or IFN-Y) only. Online supplemental appendix I lists the full search strategy for MEDLINE. The following electronic databases will be searched: Medline (via Ovid), Embase (via Elsevier), Cochrane Library (via Wiley, including Cochrane Central Register of Controlled Trials) and Web of Science Core Collection (via Clarivate Analytics, including Science Citation Index Expanded and Social Sciences Citation Index). The reference list of all studies selected for critical appraisal will be screened for additional studies. There will be no restrictions or limits on the age or sex of subjects. The language will be restricted to English. Filters will be used to limit results to human studies (online supplemental table 1).

\section{Study selection}

After the search, all identified studies will be uploaded to Endnote X9 (Clarivate Analytics, Pennsylvania, USA) and Covidence. ${ }^{17}$ We will remove duplicate studies. There will be two levels of screening. First, two reviewers (OS and AM) will independently screen the titles and abstracts of studies for eligibility and data extraction. Next, the two reviewers will then independently evaluate the full-text articles for inclusion/exclusion. Should discrepancies arise, the two reviewers will resolve the disagreements by discussion. Should that fail to be resolved, (AG) will reconcile disagreements between the two reviewers through expert advice. Through Covidence, we will generate a PRISMA document with the number of results we started with, the number excluded during title/abstract screening, and the number excluded during full-text assessments, along with reasons for exclusion. We will also manually include preselected studies that we consider important, and that were not captured during the search. We will include the final number in the final report.

\section{Data extraction}

Data will be extracted using a standardised extraction form. Extracted data will consist of: type of study, number of participants, study methods, the technology used to measure the three proinflammatory cytokines (TNF, IL-1 $\beta$ and IFN- $\gamma$ ), and outcomes of significance as described above. We will also extract clinical factors in the patient cohorts examined. Such factors might include age, comorbidities, gender, race, body mass index, HIV infection, other forms of immunosuppression (chemotherapy, radiation, leukaemia and lymphoma), type of sepsis (bacterial, viral, fungal, etc), sepsis severity (sepsis, severe sepsis and septic shock), the site of origin of the infection (blood, urine, skin and soft tissue, respiratory, other). The level of organ dysfunction in the cohorts will 
be assessed by recording the SOFA score during the first 24 hours after admission.

Study data will be collected and managed using Research Electronic Data Capture tools hosted at the University of Colorado Denver. Additional relevant information that arises during data extraction will be recorded, and this will be reported in the final systematic review manuscript. Two independent persons (OS and AM) will be involved with data extraction. Disagreements will be resolved by the assessment of a third reviewer (AG). All extraction forms, tools, and meta-analyses will be pilot tested in two studies and subsequently modified to ensure the extraction of relevant data and assess the actual feasibility of our research question. If any data are missing from a study or should uncertainties arise, we will attempt to contact the study authors to obtain the missing data or clarify any ambiguity.

\section{Quality assessment}

Two reviewers (OS and AM) will independently evaluate the included studies for risk of bias, and any disagreements will be resolved by (AG). The methodological quality of included studies will be assessed independently by the same two reviewers according to the Quality in Prognosis Studies (QUIPS) tool. ${ }^{18}$ The QUIPS tool considered 6 domains of bias: study participation, study attrition, prognostic factor measurement, outcome measurement, study confounding and statistical analysis and reporting. Each domain is composed of a list of items that enable assessment of the domain as low, moderate or high risk. If the majority of criteria were met, a '++' rating was given. If some of the criteria were met, a ' + ' rating was given. If most of the criteria is not met, studies were rated as low quality, and they were excluded.

\section{Risk of bias}

We will produce a funnel plot to assess for publication bias. Studies with high precision will be plotted closer to the average, and those with low precision will spread on either side of the average, producing a funnel-shaped distribution. The more asymmetric the shape is, the more profound the amount of bias in the meta-analysis is. ${ }^{19}$

\section{Data synthesis}

Studies will, where possible, be pooled in a statistical meta-analysis. All data will be presented and synthesised through tables' summaries, figures and charts, where appropriate. Quantitative data will be pooled with statistical meta-analysis. Effect sizes will be expressed as ORs or risk ratios for dichotomous data and weighted mean differences (for continuous data), and their $95 \%$ CIs will be calculated. We will report study level variables, and they will be evaluated by their mean or proportion. Study level heterogeneity will be assessed using the I-square test. We plan to do a fixed or random effect meta-analysis method depending on the studies' heterogeneity and sample size.

Exploratory subgroup analyses will be performed, where possible, based on the following:
- Studies mean age continuous or dichotomised using the mean.

- Studies primary cytokine measurement technique (ELISA vs immunological/magnetic beads).

- Year of the study publication, as continuous.

- Type of studies (prospective, case-control, crosssectional or randomised clinical trials).

- The proportion of studies participants per immune function status (Immunocompetent vs immunocompromised) or comorbidities (congestive heart failure or malignancy; yes or no).

- Cohort sample size, as continuous or dichotomised using the mean.

- Studies proportion of gender; as continuous or dichotomised using the mean.

- Studies proportions of the type of sepsis source included.

- Studies proportions of sepsis severity.

- Studies with the inclusion of a specific sepsis source (respiratory vs not; abdominal sepsis vs not, urinary sepsis vs not, etc).

- Studies mean SOFA scores; as continuous or dichotomised using the mean

Continuous variables extracted means will be checked for linearity using a two-way scatter plot against logtransformed cytokine mean levels and a coefficient correlation using meta-regression.

\section{Assessing certainty in the findings}

We will follow the Grading of Recommendations Assessment, Development and Evaluation (GRADE) system for quantifying the quality of evidence. A Summary of Findings (SoF) will be created using GRADEPro Guideline Development Tool (GDT) as feasible. All primary outcomes will be included in the SoF. The SoF will present the following information, where appropriate: a ranking of the quality of the evidence based on the risk of bias, directness, heterogeneity, precision and risk of publication bias of the review results. Summary results from meta-analyses will be reported as summary point estimates and interval estimates. The meta-analysis forest plots will be presented as appropriate. If used, results of a funnel plot for publication bias or risk of publication bias will be presented. If meta-analysis is not performed, a narrative summary in words and tables will be included.

\section{ETHICS AND DISSEMINATION}

Ethical approval is not required for this systematic review since it aims to collect data from existing literature. As such, there are no privacy concerns. Findings will be disseminated in the form of peer-reviewed publications and abstract presentations at research conferences.

In conclusion, our review will evaluate the available evidence for key cytokine levels and their association with sepsis outcomes using the GRADE approach. ${ }^{20}$ We believe that this work will address an important gap by reporting on a gold-standard reference for cytokine levels in sepsis. 
This work will also address the concept of 'cytokine storm' in sepsis. Our findings may guide future sepsis management strategies, such as cytokine-blocking therapies.

\section{Author affiliations}

${ }^{1}$ Department of Internal Medicine, American University of Beirut, Beirut, Lebanon

${ }^{2}$ Yale School of Medicine, Department of Internal Medicine, Waterbury Hospital,

Waterbury, Connecticut, USA

${ }^{3}$ School of Medicine, University of Colorado Anschutz Medical Campus, Aurora,

Colorado, USA

${ }^{4}$ Department of Medicine, University of Colorado Anschutz Medical Campus, Aurora,

Colorado, USA

${ }^{5}$ Health Sciences Library, University of Colorado Denver, Aurora, Colorado, USA

${ }^{6}$ Neurolgy, University of Colorado Anschutz Medical Campus, Aurora, Colorado, USA

${ }^{7}$ Department of Medicine, Hospital Infantil de México Federico Gomez, Mexico City,

Mexico City, Mexico

${ }^{8}$ Department of Medicine, Rocky Mountain Regional VA Medical Center, Aurora,

Colorado, USA

Twitter Amal Gharamti @AmalGharamti and Andrés Henao-Martínez @andhen25

Contributors AM designed the systematic review protocol with the help of the librarian $K D$. AG and LS prepared the first draft of the manuscript. OS, AH-M, SS, $\mathrm{KD}, \mathrm{SS}, \mathrm{CF}-\mathrm{P}, \mathrm{AH}-\mathrm{M}$ and LS reviewed and revised the first draft. All authors read and approved the manuscript.

Funding This study was supported by grant UL1 RR025780 from the National Institutes of Health/National Centre for Research Resources, Colorado Clinical Translational Science Institute. L.S. is funded by the Emily Foundation for Medical Research.

Competing interests None declared.

Patient consent for publication Not required.

Provenance and peer review Not commissioned; externally peer reviewed.

Supplemental material This content has been supplied by the author(s). It has not been vetted by BMJ Publishing Group Limited (BMJ) and may not have been peer-reviewed. Any opinions or recommendations discussed are solely those of the author(s) and are not endorsed by BMJ. BMJ disclaims all liability and responsibility arising from any reliance placed on the content. Where the content includes any translated material, BMJ does not warrant the accuracy and reliability of the translations (including but not limited to local regulations, clinical guidelines, terminology, drug names and drug dosages), and is not responsible for any error and/or omissions arising from translation and adaptation or otherwise.

Open access This is an open access article distributed in accordance with the Creative Commons Attribution Non Commercial (CC BY-NC 4.0) license, which permits others to distribute, remix, adapt, build upon this work non-commercially, and license their derivative works on different terms, provided the original work is properly cited, appropriate credit is given, any changes made indicated, and the use is non-commercial. See: http://creativecommons.org/licenses/by-nc/4.0/.

ORCID iDs

Amal Gharamti http://orcid.org/0000-0002-6175-299X
Carlos Franco-Paredes http://orcid.org/0000-0001-8757-643X

Andrés Henao-Martínez http://orcid.org/0000-0001-7363-8652

Leland Shapiro http://orcid.org/0000-0002-1392-3475

\section{REFERENCES}

1 Rudd KE, Johnson SC, Agesa KM, et al. Global, regional, and national sepsis incidence and mortality, 1990-2017: analysis for the global burden of disease study. Lancet 2020;395:200-11.

2 Torio C, Moore B. National inpatient hospital costs: the most expensive conditions by payer, 2013. HCUP statistical brief No. 204. 2017. Rockville, MD: Agency for Healthcare Research and Quality, 2016.

3 Bone RC, Sibbald WJ, Sprung CL. The ACCP-SCCM consensus conference on sepsis and organ failure. Chest 1992;101:1481-3.

4 Singer M, Deutschman CS, Seymour CW, et al. The third International consensus definitions for sepsis and septic shock (Sepsis-3). JAMA 2016;315:801-10.

5 Jekarl DW, Kim JY, Ha JH, et al. Diagnosis and prognosis of sepsis based on use of cytokines, chemokines, and growth factors. Dis Markers 2019;2019:1-11.

6 Huang M, Cai S, Su J. The pathogenesis of sepsis and potential therapeutic targets. Int J Mol Sci 2019;20:5376.

7 Chousterman BG, Swirski FK, Weber GF. Cytokine storm and sepsis disease pathogenesis. Semin Immunopathol 2017;39:517-28.

8 Tisoncik JR, Korth MJ, Simmons CP, et al. Into the eye of the cytokine storm. Microbiol Mol Biol Rev 2012;76:16-32.

9 Ferrara JL, Abhyankar S, Gilliland DG. Cytokine storm of graftversus-host disease: a critical effector role for interleukin-1. Transplant Proc 1993;25:1216-7.

10 Imashuku S. Clinical features and treatment strategies of EpsteinBarr virus-associated hemophagocytic lymphohistiocytosis. Crit Rev Oncol Hematol 2002;44:259-72.

11 Jahrling PB, Hensley LE, Martinez MJ, et al. Exploring the potential of variola virus infection of cynomolgus macaques as a model for human smallpox. Proc Natl Acad Sci U S A 2004;101:15196-200.

12 Abraham E, Anzueto A, Gutierrez G, et al. Double-Blind randomised controlled trial of monoclonal antibody to human tumour necrosis factor in treatment of septic shock. NORASEPT II study Group. Lancet 1998;351:929-33.

13 Bone RC. Why sepsis trials fail. JAMA 1996;276:565-6.

14 Marik PE, Khangoora V, Rivera R, et al. Hydrocortisone, vitamin C, and thiamine for the treatment of severe sepsis and septic shock: a retrospective before-after study. Chest 2017;151:1229-38.

15 Moher D, Shamseer L, Clarke M, et al. Preferred reporting items for systematic review and meta-analysis protocols (PRISMA-P) 2015 statement. Syst Rev 2015;4:1.

16 Moher D, Altman DG, Liberati A, et al. PRISMA statement. Epidemiology 2011;22:128.

17 Innovation V. Covidence systematic review software. Melbourne, Australia, 2017.

18 Hayden JA, van der Windt DA, Cartwright JL, et al. Assessing bias in studies of prognostic factors. Ann Intern Med 2013;158:280-6.

19 Higgins JP, Thomas J, Chandler J. Cochrane Handbook for systematic reviews of interventions. John Wiley \& Sons, 2019.

20 Guyatt G, Oxman AD, Akl EA, et al. Grade guidelines: 1. IntroductionGRADE evidence profiles and summary of findings tables. J Clin Epidemiol 2011;64:383-94. 